\title{
ORIGINAL
}

\section{DISEÑO Y VALIDACIÓN DE DOS ESCALAS PARA MEDIR LA ACTITUD HACIA LA ALIMENTACIÓN Y LA ACTIVIDAD FÍSICA DURANTE LA ADOLESCENCIA}

\author{
Marta Lima-Serrano (1), Joaquín Salvador Lima-Rodríguez (1) y África Sáez-Bueno (2). \\ (1) Departamento de Enfermería. Facultad de Enfermería, Fisioterapia y Podología. Universidad de Sevilla. \\ (2) Centro de Salud de Montequinto. Distrito Sanitario Sevilla Sur. Servicio Andaluz de Salud.
}

No existe ningún conflicto de interés.

\section{RESUMEN}

Fundamentos: Diversos autores proponen la actitud como un mediador en el cambio de comportamiento, por lo que puede predecir la práctica de este. El objetivo del trabajo fue diseñar y validar dos escalas para medir la actitud de los adolescentes hacia la alimentación saludable, y la actitud hacia la actividad física.

Métodos: Se realizó el diseño tras una revisión de la literatura. Se procedió a la validación mediante un panel Delphi on-line con 18 expertos/as, un pretest, y una prueba piloto con una muestra de 188 alumnos/as de educación secundaria. Se analizaron la comprensibilidad, la validez de contenido, la adecuación, la fiabilidad mediante la prueba alpha de Cronbach, y la validez de constructo, mediante el análisis factorial exploratorio.

Resultados: Las escalas validadas por los expertos/as se consideraron adecuadas en el pretest. En la prueba piloto, la Escala de Actitud hacia la Alimentación de diez ítems, obtuvo un $\alpha=0,72$. La Escala de Actitud hacia la Actividad Física de ocho ítems, tuvo un $\alpha=0,86$. En el análisis factorial mostraron datos a favor de su unidimensionalidad, a) todos los ítems obtuvieron pesos $r>0,30$ en el primer factor sin rotar, $b$ ) este explicaba una proporción importante de varianza con respecto a los demás, c) la varianza total explicada por los factores principales era mayor al $50 \%$.

Conclusiones: Las escalas demostraron su validez y fiabilidad y pueden ser utilizadas para valorar la actitud hacia estas áreas de atención prioritarias en adolescentes españoles, y para evaluar este resultado intermedio de intervenciones y programas de salud.

Palabras clave: Andalucía. Adolescencia. Actitud hacia la salud. Nutrición Adolescente. Actividad Física. Escala. Estudios de Validación.

\section{Correspondencia}

Marta Lima Serrano.

Departamento de Enfermería

Facultad de Enfermería, Fisioterapia y Podología

Universidad de Sevilla

Correo electrónico: mlima@us.es

\section{ABSTRACT \\ Design and Validation of Scales to Measure Adolescent Attitude toward Eating and toward Physical Activity}

Background: Different authors suggest that attitude is a mediator in behavior change, so it is a predictor of behavior practice. The main of this study was to design and to validate two scales for measure adolescent attitude toward healthy eating and adolescent attitude toward healthy physical activity.

Methods: Scales were design based on a literature review. After, they were validated using an on-line Delphi Panel with eighteen experts, a pretest, and a pilot test with a sample of 188 high school students. Comprehensibility, content validity, adequacy, as well as the reliability (alpha of Cronbach test), and construct validity (exploratory factor analysis) of scales were tested.

Results: Scales validated by experts were considered appropriate in the pretest. In the pilot test, the ten-item Attitude to Eating Scale obtained $\alpha=0.72$. The eight-item Attitude to Physical Activity Scale obtained $\alpha=0.86$. They showed evidence of one-dimensional interpretation after factor analysis, a) all items got weights $r>0.30$ in first factor before rotations, b) the first factor explained a significant proportion of variance before rotations, and c) the total variance explained by the main factors extracted was greater than $50 \%$.

Conclusions: The Scales showed their reliability and validity. They could be employed to assess attitude to these priority intervention areas in Spanish adolescents, and to evaluate this intermediate result of health interventions and health programs.

Key words: Adolescent. Attitude. Adolescent Nutrition. Physical Activity. Scale. Validation Studies. 


\section{INTRODUCCIÓN}

La obesidad es considerada una epidemia en el siglo XXI ${ }^{1}$. Presenta una ascendente prevalencia, fundamentalmente en los países industrializados, supone un factor de riesgo para diversas enfermedades (diabetes, enfermedades cardiovasculares, artrosis, cáncer, etc.) y genera elevados costes socioeconómicos ${ }^{2}$. En España, se calculó que en el año 2002 los costes asociados a la obesidad representaron alrededor del $7 \%$ del gasto sanitario total ${ }^{3}$.

El aumento en las dietas de azúcares libres y de grasas saturadas, unido a la disminución de la actividad física, ha influido en esta situación que afecta en primera línea a la población adolescente ${ }^{4}$, también muy vulnerable a los problemas de imagen corporal, debido a cambios fisiológicos, emocionales, cognitivos y sociales, que provocan una mayor preocupación por la apariencia física ${ }^{5}$. Estar delgado/a se ha convertido en un gran valor social y la estigmatización, debida al sobrepeso y obesidad, está incrementando ${ }^{6,7}$. La imagen corporal influye en el autoconcepto y en la salud mental y bienestar psicológico y está influida por situaciones de sobrepeso y obesidad $^{7-10}$.

Recientemente, el estudio internacional Health Behaviour in School Age Children (HBSC), dirigido por la Organización Mundial de la Salud (OMS) ${ }^{11}$, encontró que el 14,3\% de los/as adolescentes encuestados se encontraban en situación de sobrepeso y obesidad, medidos como índice de masa corporal (IMC) en el decil 85 o superior. En adolescentes españoles estos datos son más elevados $(16,8 \%)$. En cuanto a la imagen corporal, mostró que el $28,3 \%$ se veía "demasiado gordo/a", obteniéndose puntuaciones más altas en España (30,8\%).
La ausencia de desayuno está relacionada con la realización de dietas no saludables, incrementando el consumo de snacks bajos en fibras y ricos en grasas saturadas, lo que incrementa el riesgo de obesidad. El HBSC encontró que el $37,7 \%$ de los/as adolescentes encuestados no desayunaba los cinco días de actividad escolar. En España el porcentaje fue $34,8 \%$. En cuanto al consumo de azúcares libres, el $22 \%$ de los/as adolescentes de este estudio consumía bebidas azucaradas a diario, siendo los resultados similares en España ${ }^{11}$. Esto significa un descenso en el consumo de estas bebidas, tanto en los datos internacionales, como nacionales ${ }^{12}$. En España, en 2006, el 37\% las consumía entre cinco y siete veces a la semanas, mientras que el 31,3\% afirmaba comer dulces (caramelos, chocolate) de cinco a siete veces a la semana ${ }^{12,13}$. En cambio, para el resto de comportamientos alimentarios mostrados, así como para el IMC, los resultados de la actual edición fueron más desfavorables que los encontrados en la edición de $2006^{12}$.

La práctica de actividad física en niños $y$ adolescentes es cada vez menos frecuente, lo que conlleva a que el sedentarismo se haya convertido en un problema de Salud Pública ${ }^{15}$. En un estudio realizado en España, en adolescentes de entre 12 y 18 años, se detectó que 25,3\% eran inactivos o sedentarios $^{16}$. La OMS recomienda que los/as adolescentes acumulen un mínimo de 60 minutos diarios de actividad física, mayoritariamente aeróbica, de intensidad moderada o vigorosa. Asimismo, sería conveniente que realizaran tres veces por semana, como mínimo, prácticas de actividades que fortalezcan el aparato locomotor ${ }^{15}$. Sin embargo, en el estudio HBSC se encontró que sólo el 19\% seguía esta recomendación, y en España el 23,7\% ${ }^{11}$. 
Se ha encontrado también relación entre la adopción de conductas de riesgo para la salud durante la adolescencia y el estilo sedentario. Por tanto la actividad física regular y el mantenimiento de un peso corporal adecuado son elementos potencialmente importantes para evitar la aparición temprana de este tipo de comportamientos ${ }^{7}$.

El abordaje de esta situación requiere estrategias de promoción de la salud y prevención del riesgo. En España, la respuesta fue la puesta en marcha de la Estrategia NAOS (Nutrición, Actividad física, prevención de la Obesidad y Salud) ${ }^{17}$, y la creación del programa PERSEO ${ }^{18}$ dirigido de manera prioritaria a la intervención en el medio escolar, con el objetivo de que niños y jóvenes adopten este tipo de hábitos de alimentación saludable, junto con la estimulación de la práctica de deporte y actividad física ${ }^{3}$. En Andalucía, el Plan para la Promoción de la Actividad Física y la Alimentación Equilibrada, entre las acciones centradas en la población adolescente, destaca la elaboración de materiales educativos y de difusión, la organización de distintas actividades formativas, el impulso de actividad física en los centros educativos y de hábitos alimenticios $\operatorname{sanos}^{19}$.

La evaluación de estas estrategias y programas es una necesidad institucional, ya que se debe saber cuáles son eficaces y ayudan realmente a los/as ciudadanos ${ }^{20}$. La evaluación puede ser del proceso o del efecto o impacto de un programa permitiendo comprobar las mejoras alcanzadas en la población como resultado de la intervención ${ }^{21}$. Dado que diversos modelos teóricos sobre el cambio de comportamientos proponen la actitud como un mediador cognitivo de dicho cambio, la evaluación podría traducirse en determinar si se consigue que los adolescentes desarrollen actitudes favorables hacia la alimentación y la actividad física como predictoras de prácticas saludables relacionadas con estas áreas ${ }^{22-25}$.
Con frecuencia, cuestionarios, test o escalas son utilizados en la evaluación de la alimentación, el ejercicio físico o de constructos considerados mediadores de comportamientos relacionados con estas áre$a^{15}$. En la bibliografía se pueden encontrar escalas para valorar el consumo de alimen$\operatorname{tos}^{12,26}$, la actividad y el ejercicio físico en adolescentes $^{12,27,28}$, así como los conocimientos, las preferencias alimentarias, los determinantes de la dieta ${ }^{26,29,30}$, el autoconcepto físico ${ }^{29}$ y la intencionalidad para ser físicamente activo ${ }^{32}$. Sin embargo, no se localizó ninguna específica y válida para la población española que permitiera hacer una valoración global de la actitud de los/as adolescentes hacia la alimentación y la realización de actividad y ejercicio físico, desde la perspectiva de su acción sobre la salud.

El objetivo de este trabajo ha sido construir y validar dos escalas que permitan valorar la actitud hacia la alimentación saludable y la actitud hacia la actividad física en adolescentes.

\section{SUJETOS Y MÉTODOS}

Se realizó el diseño a partir de la revisión bibliográfica $\mathrm{y}$, posteriormente, se procedió a la validación de las dos escalas mediante el consenso de expertos, un pretest, y una prueba piloto para el análisis de sus propiedades psicométricas (fig. 1) ${ }^{33}$.

\section{Diseño de las escalas}

\section{1) Elección de las actitudes a estudiar.} Se revisaron bases de datos nacionales e internacionales (Pubmed, Scopus, Índice Médico Español, Cuiden Plus, Google Académico) y se consultaron páginas web institucionales (Ministerio de Sanidad, Servicios Sociales e Igualdad, Organización Mundial de la Salud, entre otras), para clarificar la definición del rasgo o actitud que se pretendía medir e identificar instru- 
mentos utilizados en adolescentes ${ }^{34}$. Las palabras clave usadas en español y traducidas al inglés usando el tesauro propuesto por Pubmed (Mesh), con sus posibles variaciones, fueron: adolescencia o jóvenes, y valoración o escalas o cuestionarios, $\mathrm{y}$ actitud, y alimentación o actividad física.

Se consideró que el sujeto adolescente con actitud alta (o favorable) sería quien valora: a) la necesidad y los beneficios de realizar una alimentación sana y equilibrada, evitando las situaciones perjudiciales con respecto a la alimentación, y b) la necesidad y los beneficios de la actividad física para la salud. El sujeto adolescente con baja actitud (o desfavorable) sería aquel o aquella que no valora: a) la necesidad de una alimentación sana ni sus beneficios, prefiriendo consumir alimentos inadecuados, y b) la necesidad de realizar actividad física ni los beneficios de la misma para la salud.

\section{2) Selección de ítems y elaboración de} las escalas. En base a la teoría y mediante la recopilación de los instrumentos de medida publicados, se obtuvo un conjunto de ítems que aludían a las dimensiones propuestas para las actitudes hacia la alimentación saludable y la actividad física $^{12,19,26,29,35-37}$. Se contó también con instrumentos facilitados por el grupo GENUD (Growth, Exercise, NUtrition and Development $)^{38}$, con el que se contactó tras conocer su relación con el estudio HELENA (Healthy Lifestyle in Europe by Nutrition in Adolescence $)^{39}$. Finalmente el equipo diseñó preguntas específicas para las dimensiones definidas y que no estaban bien representadas en el material consultado. Como punto de partida, sobre el número de ítems necesario se estimó que a mayor número de ellos sería más fácil hacer una buena selección final ${ }^{34}$. Se eligió una escala Likert de cinco puntos, que permite dar un grado o intensidad al enunciado de cada ítem. De esta forma se construyó la primera versión de las escalas con un total de 40 ítems, 25 para la Escala de Actitud hacia la Alimentación y 15 para la Escala de Actitud hacia la Actividad Física.

\section{Validación}

1) Validez de contenido. Entre mayo y julio de 2010 se realizó un panel Delphi ${ }^{40}$ on-line con 40 personas expertas del sector de la salud, relacionadas con la atención sanitaria y la promoción de hábitos saludables en adolescentes y seleccionadas mediante muestreo intencional, para la depuración y el refinamiento de la escala inicial. Se les informó de los objetivos del estudio y se les invitó a participar voluntariamente, garantizando la confidencialidad y privacidad de sus aportaciones. En la primera ronda respondieron 18 personas. En la segunda ronda respondieron 17 personas.

$11(61 \%)$ participantes eran mujeres. El nivel académico máximo alcanzado fue de diplomado por $8(44 \%)$ participantes, licenciado por $3(17 \%)$ y de doctor o diploma de estudios avanzados por 7 (39\%). 9 (50\%) participantes se dedicaban a la docencia, $4(22 \%)$ a la práctica clínica y $4(22 \%)$ a la gestión. $6(33 \%)$ habían publicado artículos relacionados con la temática.

En el formulario enviado, junto a las instrucciones para su cumplimentación, se incluía una definición sobre el constructo a evaluar, por cada una de las escalas. Se les solicitaba que valoraran de 1 a 5 el grado de adecuación de cada uno de los ítems propuestos para el instrumento inicial. Mediante respuestas abiertas, podían sugerir nuevos ítems o modificar los propuestos.

Tras completar cada ronda, las respuestas fueron analizadas teniendo en cuenta 


\section{Figura 1}

\section{Esquema del proceso de desarrollo de los cuestionarios}
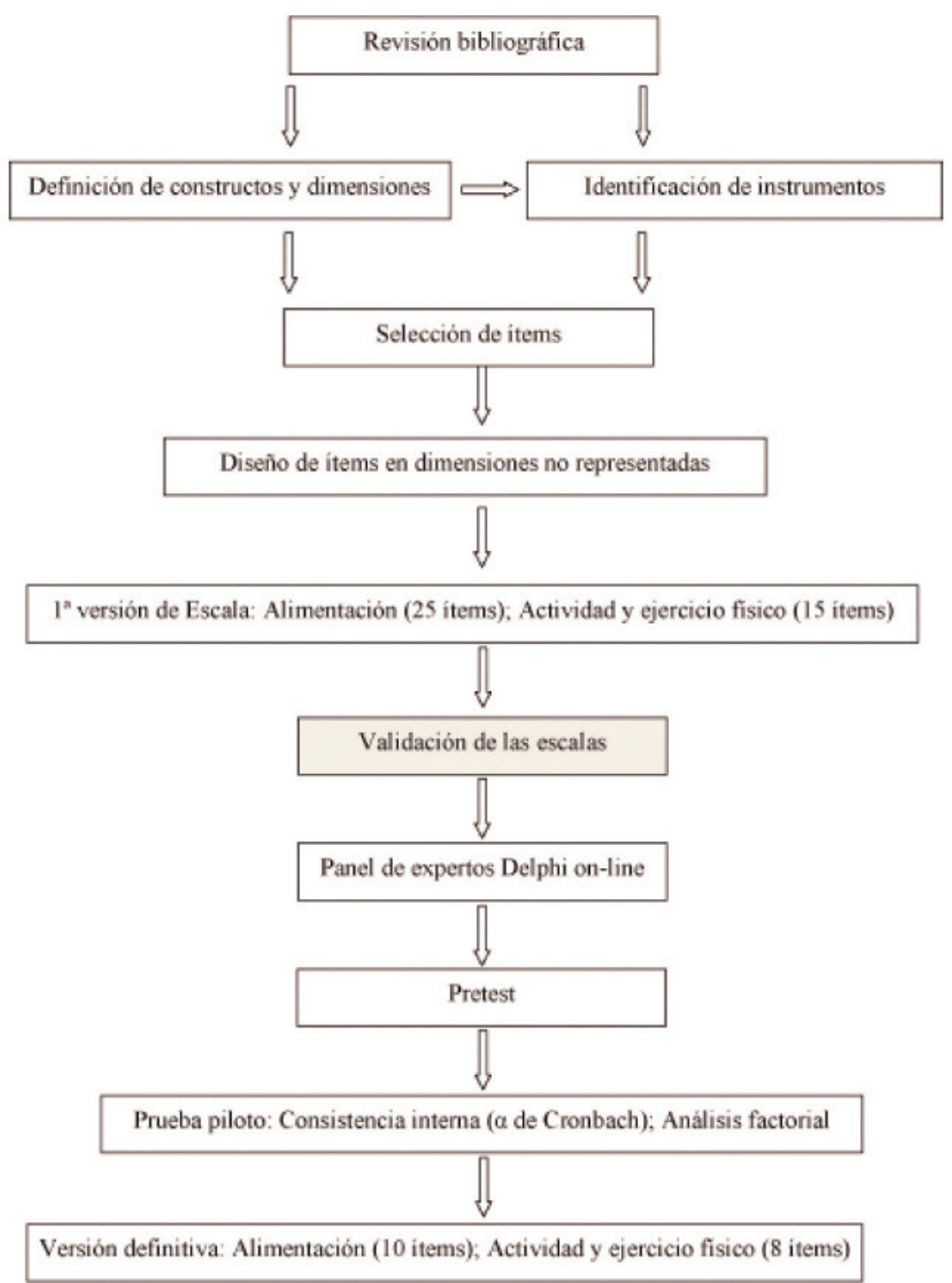
los siguientes criterios: para desestimar un ítem: media $<3,5$; mediana $<3$; para aceptar un ítem: valoraciones altas $(4-5)>75 \%$ y/o desviación típica $<0,90^{41}$. Los ítem que no habían sido ni rechazados ni aceptados pasaban a la siguiente ronda, hasta alcanzar el consenso esperado. Se realizó un análisis de las respuestas a las preguntas abiertas, que permitió realizar modificaciones a alguno de los ítems, así como proponer nuevos ítems. Después de cada ronda se enviaba a los/as expertos/as un informe de resultados, como medida de retroalimentación. Se hicieron dos rondas de valoración.

2) Pretest. Se aplicó el cuestionario a 21 adolescentes matriculados en cuarto de Educación Secundaria Obligatoria (ESO) en un Instituto de Educación Secundaria (IES) de la provincia de Sevilla. Por medio de preguntas abiertas, se les pidió que mostraran su grado de acuerdo con la adecuación del cuestionario, su comprensión, si se sentían molestos con alguna de las preguntas y si consideraban que alguna debería modificarse. Se realizó un análisis descriptivo de las categorías emergentes.

3) Análisis de las propiedades psicométricas. Aplicando las escalas validadas por los/as expertos/as, se llevó a cabo un estudio piloto a una muestra de adolescentes de la provincia de Sevilla en forma de preguntas con cinco opciones de respuesta tipo Likert. La muestra estuvo constituida por alumnos/as matriculados en cuarto de ESO en IES de gestión pública.

El tamaño muestral se definió calculando diez participantes por cada ítem de cada una de las escalas, cumpliendo con el requisito establecido para el análisis factorial. Se estimó necesaria una muestra de 210 alumnos/as ${ }^{34}$. La unidad de muestreo fue el grupo de cuarto de ESO. Se consideró que cada grupo tendría 25 alumnos/as, por lo que se realizó una selección aleatoria por conglomerados de nueve grupos. Finalmente se contó con 188 adolescentes.
La encuesta se realizó durante los meses de octubre y noviembre de 2010 utilizando un formato autoadministrado. El anonimato, la confidencialidad y la protección de datos fueron garantizados y la administración de los cuestionarios se realizó en el contexto escolar por personal entrenado. Se solicitó el consentimiento informado a los/as participantes, que se consideraron competentes para proporcionarlo, dado el tipo de estudio y su edad.

Se determinó la fiabilidad (consistencia interna) mediante la prueba alpha de Cronbach, cuyos valores oscilan entre 0 y 1 , y se consideraron aceptables los valores $\alpha>0,70$ y buenos cuando eran $\alpha>0,80^{42}$. Se determinó la validez de constructo mediante análisis factorial exploratorio. La adecuación de la muestra se valoró mediante la aplicación de la prueba de Kaiser-Meyer-Olkin (KMO), con valores mayores a 0,5 y la prueba de Esfericidad de Bartlett, con valores significativos. Se tuvieron en cuenta los siguientes criterios para determinar la unidimensionalidad: 1) que todos los ítems tuvieran coeficiente de correlación de Pearson $>0,30$ en el primer factor durante la extracción; 2) que el primer factor explicara una proporción importante de varianza con respecto a los demás factores; 3) que la varianza total explicada por los factores principales extraídos fuera mayor al 50\% $0^{34,43}$.

Se utilizó la rotación varimax para determinar las dimensiones de cada una de las escalas, se determinaron valores relevantes para incluir un ítem en los factores ortogonales, aquellos con una $\mathrm{r}$ de Pearson $>0,40^{43}$. El análisis estadístico se realizó con el programa SPSS 18.0.

El estudio fue aprobado por el Comité de Experimentación de la Universidad de Sevilla. 


\section{RESULTADOS}

1) Validez de contenido. En la tabla 1 se presenta el análisis descriptivo de los ítems estimados por el grupo de expertos/as para la Escala de Actitud hacia la Alimentación. En la primera ronda se estimaron 9 ítems ( 1 al 9) de los $25(36 \%)$ propuestos y se desestimó 1(4\%). Teniendo en cuenta las aportaciones del panel se hicieron modificaciones y se sometieron a nueva valoración 15 ítems, se estimaron 9 (60\%) ítems (10 al 21) de los propuestos. No se desestimó ningún ítem. Tras esta ronda se dio por finalizado el panel Delphi, pues 21 ítems eran suficientes para ser sometidos al estudio piloto.

Respecto a la Escala de Actitud hacia la Actividad Física (tabla 2), en la primera ronda se estimaron 10 ítems (1 al 10) de los $15(66,7 \%)$ propuestos y se desestimaron 2 (13,3\%). Al igual que en la escala anterior se hicieron modificaciones y se sometieron a nueva valoración 5 ítems que finalmente fueron estimados.

2. Valoración de la comprensión y aceptabilidad. 11 de los/as 21 adolescentes participantes en el pretest indicaron que las escalas les parecían "buenas", y 7 expresaron que las escalas eran "interesantes". Ninguno respondió a la pregunta sobre la existencia de dificultad en la comprensión de las cuestiones realizadas. Tampoco a la pregunta sobre si alguna de las cuestiones le resultaba molesta. No sugirieron modificaciones.

3) Análisis de las propiedades psicométricas. La Escala de Actitud hacia la Alimentación inicial, formada por 21 ítems, obtuvo un $\alpha=0,75$ que tras eliminar ocho ítems fue $\alpha=0,78$. Tras realizar un análisis factorial exploratorio a la versión de trece ítems, se eliminaron otros tres. La versión definitiva de diez ítems tuvo un valor $\alpha=0,72$. La Escala de Actitud hacia la Actividad Física inicial, compuesta por 15 ítems, obtuvo un $\alpha=0,79$. Tras eliminar cinco ítems resultó un valor $\alpha=0,86$. A la versión de la escala de diez elementos se le realizó un análisis factorial exploratorio que permitió eliminar otros dos. La versión definitiva de ocho ítems tuvo un valor $\alpha=0,86$.

Para la Escala de Actitud hacia la Alimentación se obtuvieron valores de $\mathrm{KMO}=0,78$ y para la Escala de Actitud hacia la Actividad Física $=0,90$. Para ambas, la prueba de Bartlett tuvo un nivel de significación menor a $<0,001$.

Se observó una agrupación de la Escala de Actitud hacia la Alimentación en torno a tres factores principales (tabla 3 ):

a) todos los ítems obtuvieron $r>0,30$ en el primer factor sin rotar.

b) El primer factor explicaba una proporción importante de varianza $(29,92 \%)$ antes de las rotaciones.

c) La varianza total explicada por los tres factores principales extraídos era de $52,9 \%$. En la rotación ortogonal varimax, los ítems obtuvieron pesos iguales o mayores a $\mathrm{r}=0,56$ en el factor al que pertenecían. La escala final puede verse en el anexo 1.

Los resultados mostraron una agrupación de la Escala de Actitud hacia la Actividad Física en torno a un factor principal (tabla 4):

a) todos los ítems tuvieron coeficientes $r>0,30$.

b) Este primer factor explicaba una proporción importante de varianza (51\%), con respecto a los demás, que explicaban el 10,46\% o menos. La escala final puede verse en el anexo 2 . 


\begin{tabular}{|c|c|c|c|c|c|c|c|c|c|c|c|c|c|c|c|c|c|c|c|c|c|c|}
\hline & f & $\hat{\infty}_{\infty}$ & $\stackrel{+}{\text { İ }}$ & ลี & $\begin{array}{l}0 \\
8 \\
8\end{array}$ & ஸे & $\tilde{m}_{\infty}$ & $\stackrel{\infty}{\stackrel{0}{=}}$ & $\stackrel{\infty}{\stackrel{1}{R}}$ & $\stackrel{\infty}{\stackrel{\infty}{\kappa}}$ & $\underset{\infty}{1}$ & ?े & ? & $\begin{array}{l}1 \\
\infty \\
\infty\end{array}$ & $\underset{\infty}{\tilde{\infty}}$ & 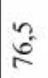 & $\begin{array}{l}\dot{\sim} \\
\infty \\
\infty\end{array}$ & $\begin{array}{l}\hat{\infty} \\
\text { in }\end{array}$ & $\begin{array}{l}n \\
2 \\
2\end{array}$ & $\begin{array}{l}n \\
2 \\
2 \\
2\end{array}$ & $\begin{array}{l}n \\
2 \\
2\end{array}$ & 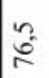 \\
\hline & en & $\underline{\hat{\sigma}}$ & $\begin{array}{l}0 \\
\text { in }\end{array}$ & $\stackrel{\infty}{\stackrel{\sim}{\sim}}$ & $\begin{array}{l}0 \\
\text { in }\end{array}$ & $\begin{array}{l}0 \\
\text { in }\end{array}$ & $=$ & $\equiv$ & $\underline{\hat{\sigma}}$ & तี & in & $\stackrel{=}{=}$ & in & 0 & 0 & in & in & m & $\stackrel{\infty}{=}$ & $\begin{array}{l}0 \\
\stackrel{0}{=}\end{array}$ & $\stackrel{\infty}{=}$ & $\stackrel{\infty}{=}$ \\
\hline$\overbrace{}^{0}$ & $I$ & 0 & 0 & 0 & $\begin{array}{l}\infty \\
\stackrel{\sim}{\sim}\end{array}$ & $\equiv$ & $\begin{array}{l}0 \\
\text { in }\end{array}$ & $\equiv$ & $\begin{array}{l}0 \\
\text { in }\end{array}$ & 0 & हे & $\stackrel{\infty}{=}$ & $\stackrel{\mathscr{0}}{=}$ & $\stackrel{\infty}{=}$ & $\stackrel{\circ}{=}$ & $\stackrel{\check{I}}{=}$ & $\stackrel{\infty}{=}$ & जे & $\stackrel{\infty}{=}$ & जे & $\stackrel{\infty}{=}$ & $\stackrel{\infty}{=}$ \\
\hline
\end{tabular}

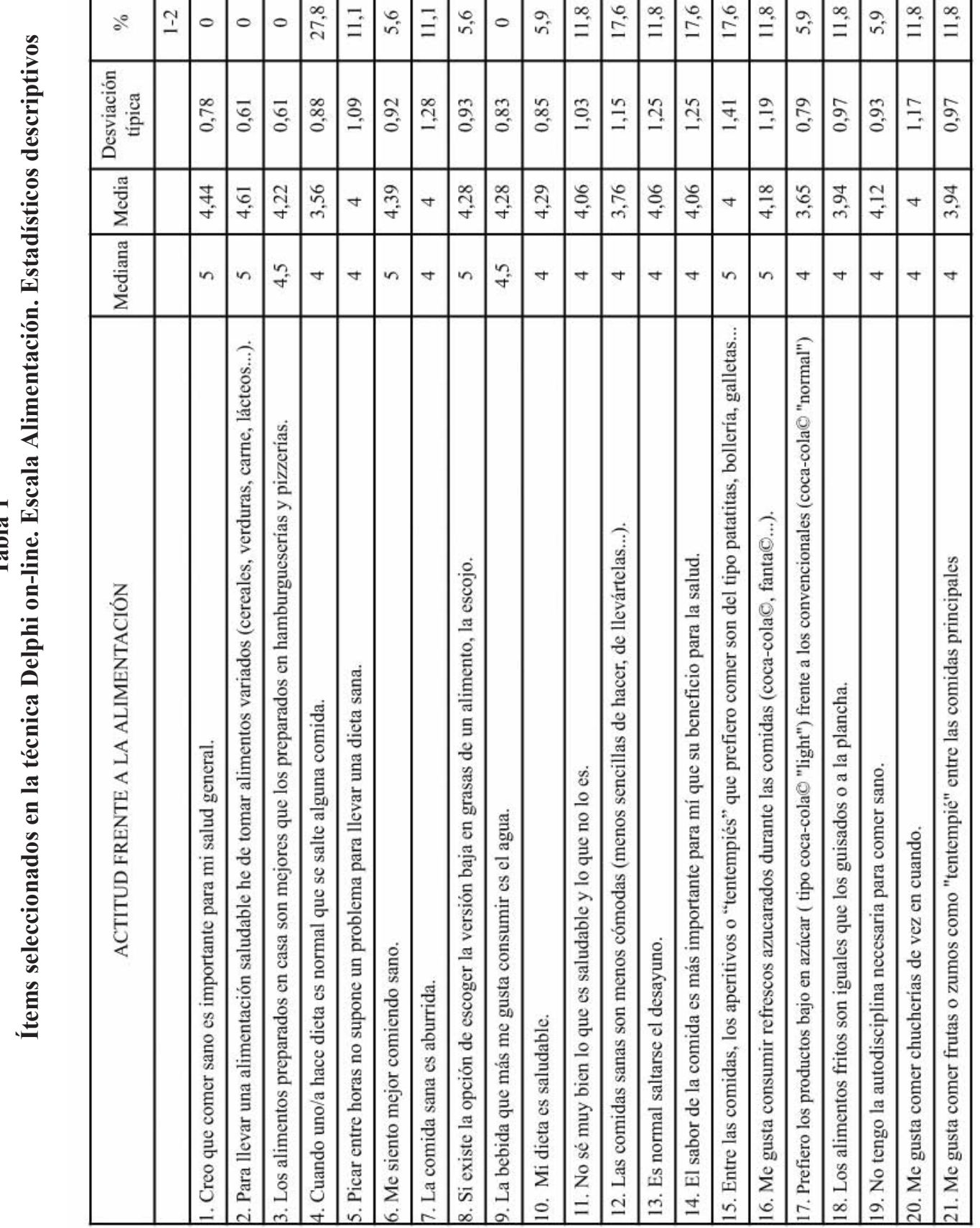




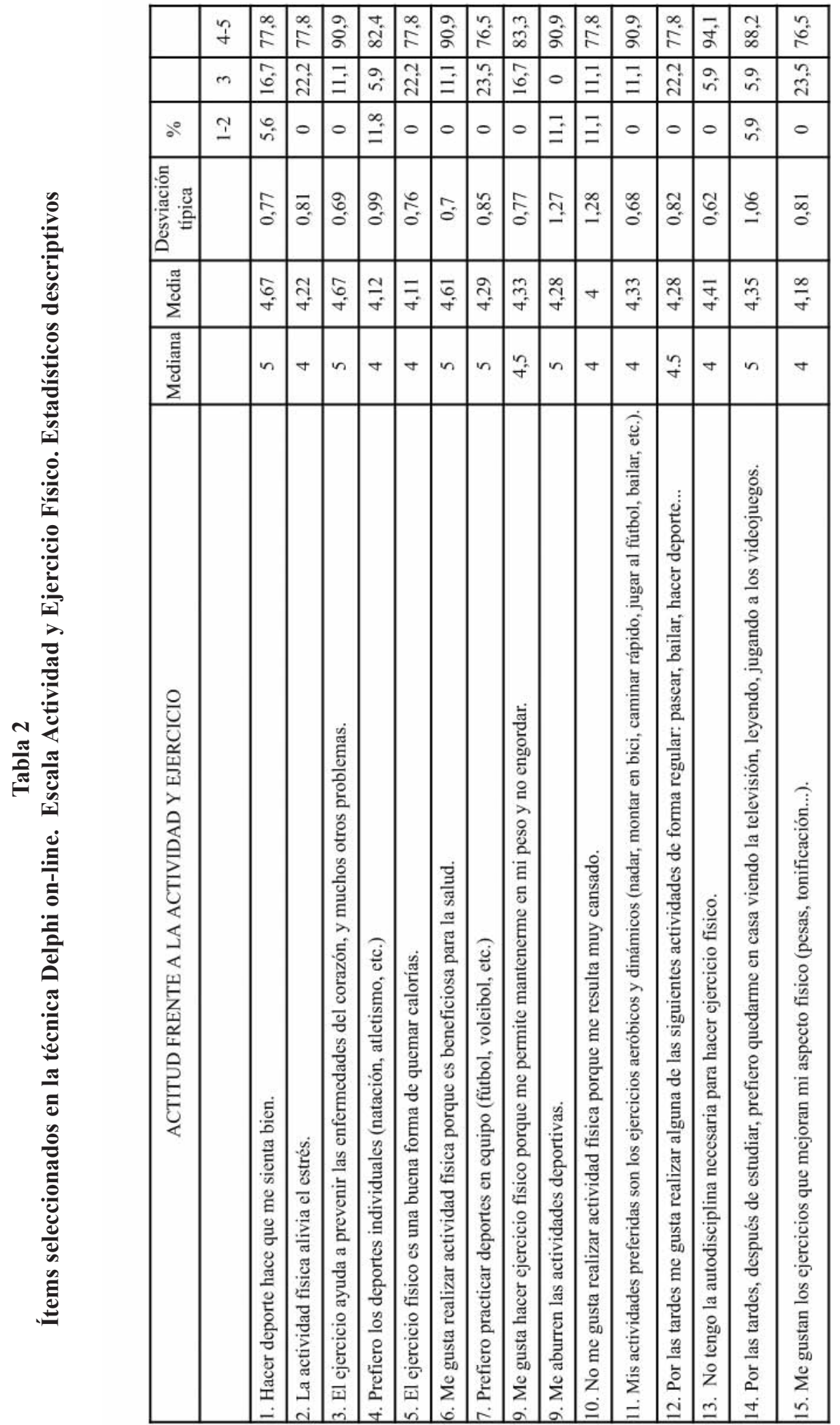




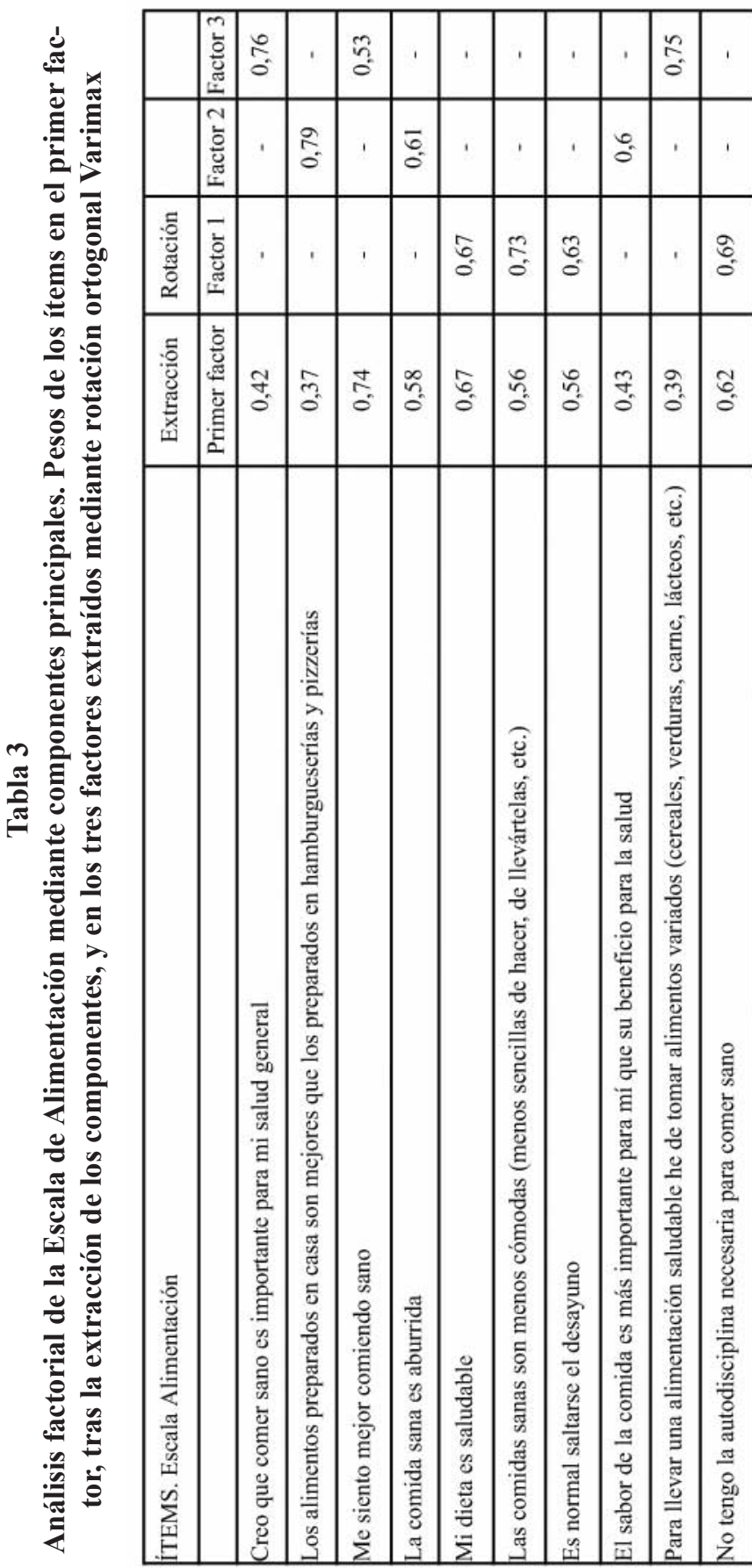


Tabla 4

Análisis factorial de la Escala de Actividad Física mediante componentes principales. Pesos de los ítems en el primer factor, tras la extracción de los componentes

\begin{tabular}{|l|c|}
\hline & $\begin{array}{c}\text { Extracción } \\
\text { Primer factor }\end{array}$ \\
\hline Hacer deporte hace que me sienta bien & 0,78 \\
\hline $\begin{array}{l}\text { Por las tardes me gusta realizar alguna de las siguientes actividades de forma regu- } \\
\text { lar: pasear, bailar, hacer deporte, etc. }\end{array}$ & 0,73 \\
\hline Me aburren las actividades deportivas & 0,82 \\
\hline La actividad fisica alivia el estrés & 0,55 \\
\hline $\begin{array}{l}\text { Por las tardes, después de estudiar, prefiero quedarme en casa viendo la televisión, } \\
\text { leyendo, jugando a los videojuegos }\end{array}$ & 0,59 \\
\hline Me gusta realizar actividad fisica porque es beneficiosa para la salud & 0,7 \\
\hline No tengo la autodisciplina necesaria para hacer ejercicio fisico & 0,68 \\
\hline No me gusta realizar actividad fisica porque me resulta muy cansado & 0,83 \\
\hline
\end{tabular}

1 componente extraido explica una varianza total: $51 \%$.

\section{DISCUSIÓN}

Diversos autores coinciden en la necesidad de diseñar y validar nuevos instrumentos de medida cuando no existan otros que midan lo que realmente pretendemos medir ${ }^{44,45}$. La técnica Delphi como método de consenso es ampliamente utilizada para la validación de escalas y cuestionarios mediante juicio de expertos ${ }^{33,40,46}$. Entre las medidas propuestas para evitar sesgos, se encuentra la estandarización de las definiciones para darlas a conocer al grupo de expertos ${ }^{43}$. También es importante explicitar los criterios de selección y que los/as expertos/as lo sean en la materia de estudio, en este caso se contó con profesionales del ámbito de la salud, relacionados con la atención y la promoción de hábitos saludables en la adolescencia ${ }^{45}$. No obstante, puede haber habido un sesgo de selección, al no tener en cuenta la opinión de profesionales expertos en Ciencias de la Actividad Física y el Deporte.

La muestra utilizada en el proceso de validación concuerda con lo propuesto en estudios previos para estabilizar las respuestas a cada uno de los ítems. El cumpli- miento de estos aspectos da mayor potencia y solidez al proceso de validación ${ }^{46}$. Los valores cuantitativos otorgados por el panel de expertos fueron elevados. Además, la introducción de un sistema que permitió realizar aportaciones cualitativas mejoró el proceso de validación ${ }^{33,46}$. A pesar de que la técnica Delphi tiene como limitación que no genera un consenso real entre los/as expertos/as, sino que los fuerza a elegir, la retroalimentación controlada puede estimular la generación de ideas y facilitar que la información irrelevante sea eliminada en el proceso de validación ${ }^{48}$.

La consistencia interna ha sido óptima en el caso de la Escala de Actitud hacia la Alimentación, y buena o alta en el caso de la Escala de Actitud hacia la Actividad Físi$\mathrm{Ca}^{38}$. El análisis de la consistencia interna mediante el alpha de Cronbach es el método más utilizado para el cálculo de la fiabilidad, al expresar este coeficiente hasta qué punto las respuestas están relacionadas entre sí, para poder concluir que todos los ítems miden lo mismo y, por lo tanto, son sumables en una puntuación total única para medir el rasgo o actitud ${ }^{34,49}$. Hay que advertir sin embargo que un alto coeficiente de 
fiabilidad no es prueba de unidimensionalidad. Es por ello, que se propuso el uso complementario del análisis factorial, que aunque supuso una ligera disminución en el alpha de Cronbach de la Escala de Actitud hacia la Alimentación, mejoró las propiedades psicométricas de la misma. El análisis factorial fue superado por las escalas presentadas, mostrando datos a favor de su interpretación unidimensional. Este se utiliza como método de validación de constructo, al permitir comprobar si se está midiendo lo que decimos que medimos, clarificando la estructura de la escala y del constructo tal como se ha definido y pretende medirse, permitiendo revisiones de la escala y su mejora $^{43,49}$.

No se puede concluir que a pesar de haber encontrado tres factores tras la rotación ortogonal en la Escala de Actitud hacia la Alimentación, la misma sea multidimensional, ya que tras una valoración racional sobre sus significados, no se encontraron conceptos que hicieran pensar en distintos subconstructos. Con frecuencia los factores pueden agrupar a los ítems con formulaciones muy parecidas, otras veces pueden reflejar peculiaridades en la formulación de los ítems, como la formulación a favor o en contra ${ }^{34,43}$. En futuros trabajos se podría ampliar la muestra para realizar el análisis factorial, ya que aunque su tamaño se acerca a la regla propuesta por diversos autores, de 10 sujetos por el número de ítems que componen la escala, resulta más estable a medida que aumenta el tamaño muestral.

Existen diversas propuestas y estrategias para la promoción de la alimentación equilibrada y la actividad física ${ }^{2,3,19}$. Entre los objetivos de estas acciones se encuentra la promoción de actitudes hacia la adopción de comportamientos saludables en relación a estas áreas de atención prioritarias, desde la consideración de que las actitudes tienen un papel mediador en la adopción de estos comportamientos ${ }^{24,25}$.
En este trabajo se presentan dos escalas que han demostrado su validez y fiabilidad. Desde el punto de vista de su aplicación práctica, estas pueden ser utilizadas en la caracterización de la población en estudios que pretendan determinar la mediación de la actitud en la adopción de hábitos alimenticios saludables y la práctica de actividad física, sirviendo también de indicadores para guiar la actuación de estrategias, programas e intervenciones, y en la evaluación del efecto sobre la actitud, como resultado intermedio de los mismos.

\section{BIBLIOGRAFÍA}

1.WHO. Diet, nutrition and the prevention of chronic diseases. Report of a Joint FAO/ WHO Expert consultation. WHO Technical report series 916. Ginebra: WHO; 2003 [consultado 21 Dic 2011]. Disponible en: http://www.who.int/dietphysicalactivity/publications/trs9 16/en/gsfao_introduction.pdf

2.Lobo F. Políticas públicas para la promoción de la alimentación saludable y la prevención de la obesidad. Rev Esp Salud Pública. 2007; 81(5): 437-441.

3.Ballesteros JM, Dal-Re M, Pérez-Farinós N, Villar C. La estrategia para la nutrición, actividad física y prevención de la obesidad (estrategia NAOS). Rev Esp Salud Pública. 2007; 81 (5): 443-449.

4.OMS. Informe sobre la salud en el mundo 2002. Ginebra: OMS; 2002 [consultado 21 Dic 2011]. Disponible en: http:/www.who.int/whr/2002/en/Chapter1S.pdf

5.Ramos P, Rivera F, Moreno C. Diferencias de sexo en imagen corporal, control de peso e índice de masa corporal de los adolescentes españoles. Psicothema. 2010; 22(1): 77-83.

6.Latner JD, Stunkard AJ. Getting worse: the stigmatization of obese children. Obes Res. 2003; 11:452-456.

7.Rodríguez-Hernández A, De la Cruz-Sánchez E, Feu S, Martínez-Santos R. Sedentarismo, obesidad y salud mental en la población española de 4 a 15 años de edad. Rev Esp Salud Pública. 2011; 85(4): 373-382.

8.Siegel JM, Yancey A, Aneshensel CS, Schuler R.. Body image, perceived pubertal timing, and adolescent mental health. J Adolesc Health. 1999; 25(2): 155-165.

9.Williams JM, Currie C. Self-esteem and physical development in early adolescence: pubertal timing and body image. J Early Adolesc, 2000; 20:129-149. 
10.Ge X, Elder GH, Regnerus M, Cox C. Pubertal transitions, perceptions of being overweight and adolescents' psychological maladjustment: gender and ethnic differences. Soc Psychol Q. 2001, 64:363-375.

11.Currie C, Zanotti C, Morgan A, Currie D, De Looze M, Roberts C, et al. (eds.). Social determinants of health and well-being among young people. Health behaviour in school-aged children (HBSC) study. International report from the 2009/2010 survey. Health policy for children and adolescents, no.6. Copenhagen: WHO Regional Office for Europe; 2012 [consultado 4 May 2012]. Disponible en: http://www.euro.who.int/ data/assets/pdf file/0003/163 $857 /$ Social-determinants-of-health-and-well-beingamong-young-people.pdf

12.Currie C, Gabhainn SN, Godeau E, Roberts C, Smith R, Currie D, et al. Inqualities in young people's health. Health behaviour in school-aged children. International report from the 2005/2006 survey. Health policy for children and adolescents, no.5. Escocia: WHO Regional Office for Europe; 2008 [consultado 18 Ene 2012]. Disponible en: http://www.euro.who.int/_data/assets/pdf_file/0005/538 52/E91416.pdf

13.Ministerio de Sanidad, Política Social e Igualdad. Desarrollo adolescente y salud en España. Resumen del estudio Health Behaviour in school Aged Children (HBSC-2006). Madrid: Ministerio de Sanidad, Política Social e Igualdad; 2011 [consultado 21 Dic 2011]. Disponible en: http://www.msc.es/profesionales/saludPublica/prevPromocion/promocion/saludJovenes/docs/D ivulgativo_completo_HBSC2006.pdf

14.Ministerio de Sanidad, Servicios Sociales e Igualdad. Resultados del Estudio HBSC-2006 en chicos y chicas españoles de 11 a 17 años. Madrid: Ministerio de Sanidad, Servicios Sociales e Igualdad; 2006 [consultado 18 Ene 2012]. Disponible en: www.msc.es/profesionales/saludPublica/prevPromocion/promocion/sa ludJovenes/docs/Tomo2_nacional2006.pdf

15.Escalante Y. Actividad física, ejercicio físico y condición física en el ámbito de la salud pública. Rev Esp Salud Pública. 2011; 84(4): 325-328

16.Cordente CA, García P, Sillero M, Domínguez J. Relación del nivel de actividad física, presión arterial y adiposidad corporal en adolescentes madrileños. Rev Esp Salud Pública. 2007; 81(3): 307-317.

17. Agencia Española de Seguridad Alimentaria. Estrategia para la nutrición, actividad física y prevención de la obesidad. Estrategia NAOS. Invertir la tendencia a la obesidad. Madrid: Ministerio de Sanidad y Consumo; 2005.

18. Ministerio de Sanidad, Servicios Sociales e Igualdad. Ministerio de Educación. Estrategia NAOS. Programa
Perseo [internet]. Madrid: Ministerio de Sanidad, Servicios Sociales e Igualdad. Ministerio de Educación [consultado 29 May 2012]. Disponible en: http://www.perseo.aesan.msssi.gob.es/es/index.shtml

19.Consejería de Salud. Plan para la promoción de la actividad física y la alimentación equilibrada. Sevilla: Junta de Andalucía [consultado 18 Ene 2012]. Disponible en: http://www.juntadeandalucia.es/salud/sites/csalud/galerias/documentos/c_1_c_6_planes_estrategias/plan_alimentacion equilibrada/Plan actividad fisica.pdf

20.Anguera MT, Chacón S, Blanco A (coord). Evaluación de programas sociales y sanitarios. Un abordaje metodológico. Madrid: Síntesis; 2008.

21.Fernández- Ballesteros R. Evaluación de programas Una guía práctica en ámbitos sociales, educativos y de salud. $2^{\text {a }}$ ed. Madrid: Síntesis; 1996.

22.Fishbein M, Ajzen I. Belief, Attitude, Intention and Behavior: An Introduction to Theory and Research. MA: Addison-Wesley; 1975.

23. De Vries H, Mudde AN. Predicting staged transitions for smoking cessation applying the attitude-social influence-autoefficacy model. Psychol Health. 1998; 13: 369-385.

24.Cerin E, Barnett A, Baranowski T. Testing Theories of Dietary Behavior Change in Youth Using the Mediating Variable Model with Intervention Programs. J Nutr Educ Behav. 2009; 41(5): 309-318.

25.Revals D, Foster D, Biddle SJH. A review of mediators of behavior in interventions to promote physical activity among children and adolescents. Preventive Medicine. 2008; 47(5): 463-470

26.Kersting M, Sichert-Hellert W, Vereecken CA, Diehl J, Be'ghin L, De Henauw S, et al. (HELENA Study Group) Food and nutrient intake, nutritional knowledge and dietrelated attitudes in European adolescents. Int J Obes (Lond). 2008; 32: S35-S41. doi:10.1038/ijo.2008.181.

27. Martínez-Gómez D, Martínez-de-Haro V, Pozo T, Welk GJ, Villagra A, Calle ME, et al. Fiabilidad y validez del cuestionario de actividad física PAQ-A en adolescentes españoles. Rev Esp Salud Pública. 2009; 83: 427-439.

28.Ortega FB, Ruiz JR, España-Romero V, VicenteRodriguez G, Martínez-Gómez D, Manios Y, et al (HELENA study group).The International Fitness Scale (IFIS): usefulness of self-reported fitness in youth. Int J Epidemiol. 2011; 40: 701-711. doi: 10.1093/ije/dyr039

29.Moreno LA, González-Gross M, Kersting M, Molna D, De Henauw S, Beghin L, et al (HELENA Study 
Group). Assessing, understanding and modifying nutritional status, eating habits and physical activity in European adolescents: The HELENA (Healthy Lifestyle in Europe by Nutrition in Adolescence) Study. Publ Health Nutr. 2008. 11(3), 288-299.

30. Vereecken C, De Henauw S, Maes L, Moreno L, Manios Y, Phillipp K, et al (HELENA Study Group). Reliability and validity of a healthy diet determinants questionnaire for adolescents. Public Health Nutr. 2009; 12: $1830-1838$.

31.Moreno JA, Cervelló E. Physical self-perception in Spanish adolescents: effects of gender and involvement in physical activity. Journal of Human Movement Studies. 2005; 48: 291-311.

32.Moreno JA, Moreno R. Cervelló EM. El autoconcepto físico como predictor de la intención de ser físicamente activo. Psicol Salud. 2007; 17(2): 261-267.

33.Carretero-Dios H, Pérez C. Standards for the development and review of instrumental studies. Int J Clin Health Psychol. 2007; 7(3): 863-882.

34.Morales P. Medición de actitudes en psicología y educación. $3^{\mathrm{a}}$ ed. Madrid: Universidad Pontificia de Comillas; 2008.

35.Margetts B M, Martinez JA, Saba A, Holm L, Kearney M. Definitions of 'healthy' eating: A pan-EU survey of consumer attitudes to food, nutrition and health. Eur J Clin Nutr. 1997; 51(Supl. 2): S23-s29.

36.Steptoe A, Pollard TM. Development of a Measure of the Motives Underlying the Selection of Food: the Food Choice Questionnaire. Appetite. 1995; 25: 267-284.

37. Lappalainen R, Kearneyc J, Gibneyc M. A Pan EU survey of consumer attitudes to food, nutrition, and health: an overview. Food Qual Prefer. 1998; 9(6): 467478.

38. Universidad de Zaragoza. GENUD-Nutrición, alimentación, crecimiento y desarrollo [internet]. Zaragoza: Universidad de Zaragoza [consultado 29 May 2012]. Disponible en: https://gestiona.unizar.es/webvrinv/datosgrupo.jsp?num_grupo $=1752$

39. HELENA. Healthy Lifestyle in Europe by Nutrition in Adolescence [internet]. Zaragoza: HELENA [consultado 29 May 2012]. Disponible en: http://www.helenastudy.com/

40.García MJ, Rodríguez F, Carmona L. Validación de cuestionarios. Reumatol Clin. 2009; 5(4): 171-177.

41.Martínez E. La Técnica Delphi como estrategia de consulta a los implicados en la evaluación de pro- gramas. Revista de Investigación Educativa. 2003; 21(2): 449-463.

42.Nunally J. Psychometric theory. New York: McGraw-Hill; 1967.

43. Morales P. El Análisis Factorial en la construcción e interpretación de tests, escalas y cuestionarios [internet]. Madrid: Universidad Pontificia Comillas; 2011 [consultado 27 Sep 2011]. Disponible en: http://www.upcomillas.es/personal/peter/investigacion/AnalisisFactorial.pdf

44.González JL, Morera A. La valoración de sucesos vitales: Adaptación española de la escala de Holmes y Rahe. Psiquis. 1983; 4(1): 7-11.

45.Moraes CL. Reichenheim ME. Cross - cultural measurement equivalence of the Revised Conflict Tactics Scales. Portuguese version used to identify violence within couples. Cad Saúde Publica. 2002 [consultado 2 Nov 2011]; 18:783-796. Disponible en: http://www.scielosp.org/pdf/csp/v18n3/9306.pdf

46.Hasson F, Keeney S, McKenna H. Research guidelines for the Delphi survey technique. J Adv Nurs. 2000; 32(4): 1008-1015.

47.Argimón JM, Jiménez J. Métodos de investigación clínica y epidemiológica. Madrid: Harcourt; 2000 .

48. Yañer R, Cuadra R. La técnica Delphi y la investigación en los servicios de salud. Cienc Enferm. 2008; 14(1): 9-15.

49. Morales P. La fiabilidad de los tests y escalas [internet]. Madrid: Universidad Pontificia Comillas; 2007 [consultado 27 Sep 2011]. Disponible en: http://www.upcomillas.es/personal/peter/estadisticabasica/Fiabilidad.pdf 


\section{Anexo 1}

\section{Escala de actitud hacia la alimentación}

Queremos conocer cuál es tu opinión sobre las siguientes cuestiones (Por favor, marca sólo una casilla para cada fila, si marcas más de una no podremos contar tu respuesta. Si es difícil elegir solo una respuesta, piensa en cuál es tu opinión la mayor parte del tiempo):

\begin{tabular}{|l|l|l|l|l|l|}
\hline ÍTEMS & $\begin{array}{c}\text { Total } \\
\text { desacuerdo }\end{array}$ & $\begin{array}{c}\text { Bastante } \\
\text { desacuerdo }\end{array}$ & $\begin{array}{c}\text { Ni acuerdo } \\
\text { ni desacuerdo }\end{array}$ & $\begin{array}{c}\text { Bastante } \\
\text { acuerdo }\end{array}$ & $\begin{array}{c}\text { Total } \\
\text { acuerdo }\end{array}$ \\
\hline Creo que comer sano es importante para mi salud general & & & & & \\
\hline $\begin{array}{l}\text { Los alimentos preparados en casa son mejores que los pre- } \\
\text { parados en hamburgueserias y pizzerias }\end{array}$ & & & & & \\
\hline Me siento mejor comiendo sano & & & & & \\
\hline La comida sana es aburrida & & & & & \\
\hline Mi dieta es saludable & & & & & \\
\hline $\begin{array}{l}\text { Las comidas sanas son menos cómodas (menos sencillas de } \\
\text { hacer, de llevártelas, etc.) }\end{array}$ & & & & & \\
\hline Es normal saltarse el desayuno & & & & & \\
\hline $\begin{array}{l}\text { El sabor de la comida es más importante para mí que su be- } \\
\text { neficio para la salud }\end{array}$ & & & & & \\
\hline No tengo la autodisciplina necesaria para comer sano & & & & & \\
\hline $\begin{array}{l}\text { Para llevar una alimentación saludable he de tomar alimentos } \\
\text { variados (cereales, verduras, carne, lácteos, etc.) }\end{array}$ & & & & & \\
\hline
\end{tabular}




\section{Anexo 2}

\section{Escala de actitud hacia la actividad física}

Queremos conocer cuál es tu opinión sobre las siguientes cuestiones (Por favor, marca sólo una casilla para cada fila, si marcas más de una no podremos contar tu respuesta. $\mathrm{Si}$ es difícil elegir solo una respuesta, piensa en cuál es tu opinión la mayor parte del tiempo):

\begin{tabular}{|c|c|c|c|c|c|}
\hline & $\begin{array}{c}\text { Total } \\
\text { desacuerdo }\end{array}$ & $\begin{array}{c}\text { Bastante } \\
\text { desacuerdo }\end{array}$ & $\begin{array}{l}\text { Ni acuerdo } \\
\text { ni desacuerdo }\end{array}$ & $\begin{array}{l}\text { Bastante } \\
\text { acuerdo }\end{array}$ & \begin{tabular}{|c} 
Total \\
acuerdo
\end{tabular} \\
\hline Hacer deporte hace que me sienta bien & & & & & \\
\hline $\begin{array}{l}\text { Por las tardes me gusta realizar alguna de las si- } \\
\text { guientes actividades de forma regular: pasear, basi- } \\
\text { lar, hacer deporte, etc. }\end{array}$ & & & & & \\
\hline Me aburren las actividades deportivas & & & & & \\
\hline La actividad física alivia el estrés & & & & & \\
\hline $\begin{array}{l}\text { Por las tardes, después de estudiar, prefiero quedar- } \\
\text { me en casa viendo la televisión, leyendo, jugando a } \\
\text { los videojuegos }\end{array}$ & & & & & \\
\hline $\begin{array}{l}\text { No me gusta realizar actividad fisica porque me re- } \\
\text { sulta muy cansado }\end{array}$ & & & & & \\
\hline $\begin{array}{l}\text { No tengo la autodisciplina necesaria para hacer } \\
\text { ejercicio fisico }\end{array}$ & & & & & \\
\hline $\begin{array}{l}\text { Me gusta realizar actividad física porque es benefi- } \\
\text { ciosa para la salud }\end{array}$ & & & & & \\
\hline
\end{tabular}

